This item was submitted to Loughborough's Institutional Repository (https://dspace.lboro.ac.uk/) by the author and is made available under the following Creative Commons Licence conditions.

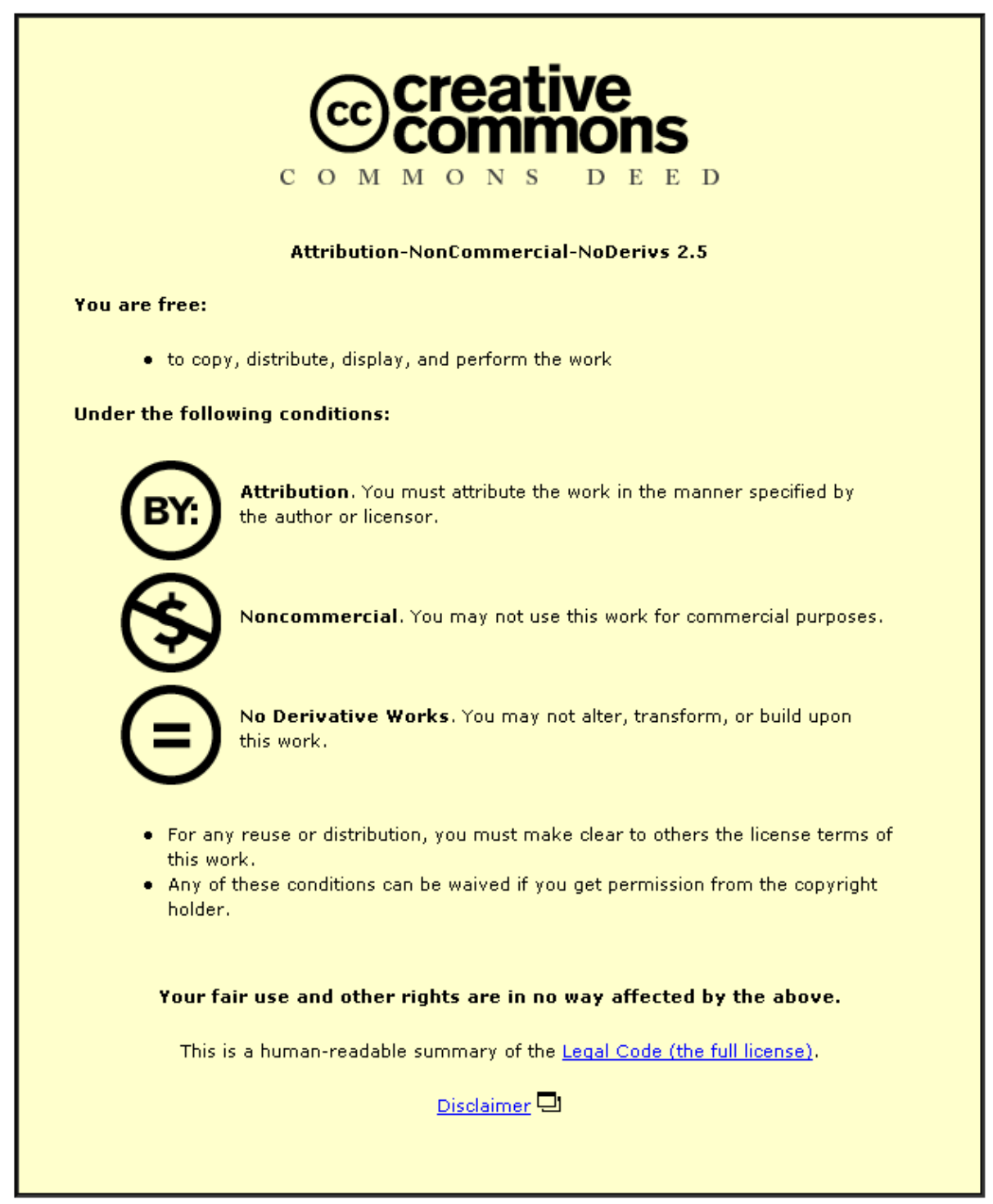

For the full text of this licence, please go to: http://creativecommons.org/licenses/by-nc-nd/2.5/ 


\title{
Facilitating B2B E-Business by IT-Supported Business Process Negotiation Services
}

\author{
Xi Chen, Paul W.H. Chung \\ Department of Computer Science, Loughborough University \\ \{x.chen, p.w.h.chung\}@lboro.ac.uk
}

\begin{abstract}
Due to the complexity of business transactions and growing business automation demands from the B2B e-business community to swiftly respond to the ever-changing environment, workflow technology has been receiving more attention recently. The increasing popularity and adoption of workflow management system (WfMS) within organisations make workflow-based B2B e-business practically viable since more and more business transactions are implemented as automated processes and executed by WfMSs. Having been viewed as services by many researchers and practitioners, process-driven B2B e-business are conducted through service discovery and runtime execution. However, if there is no existing service provided by a desired business partner that matches the requirement then such a process will have to be negotiated and then created. Unfortunately, direct people-to-people negotiation followed by manual transformation of the negotiation outcome into processdriven services can be very resource consuming. Therefore, it is identified that there is a research gap in computer-aided negotiation approach for process-driven B2B e-business. This paper introduces essentials of workflow technology and negotiation. It then describes ways of capturing elements of negotiation from an operational view point. Finally, it explains how to integrate the IT-supported negotiation services into an overall cross-organisational workflow collaboration (COWCO) supporting framework.
\end{abstract}

Keywords-workflow; business process; B2B e-business; collaboration; negotiation

\section{INTRODUCTION}

The complex and process-driven natures of business transactions as well as automation demands from business community put workflow technology as one of the potential solutions for B2B e-business. The increasing popularity and adoption of workflow management system (WfMS) within organisations make workflow-based B2B e-business practically viable. Having evolved from centrally designed $\mathrm{B} 2 \mathrm{~B}$ workflows $[1,2,3,4]$ at an early stage, the more recent development in process-driven B2B transactions has undoubtedly reaching out to service wrapping in order to suit service oriented architectures (SOA). As indicated by the works of a number of researchers and practitioners, current foci regarding B2B e-business include discovery of process-driven services $[5,6]$ and execution of distributed workflow models $[3,4]$.

This project was funded by an EPSRC research studentship through the Loughborough University Innovative Manufacturing and Construction Research Centre (IMCRC)
By following the service matchmaking and execution paradigm as found in the classic web services approach, a considerable amount of corporate assets, in the form of business transaction workflows, can be preserved, retrieved and reused in a cost-effective way. However, there is still a crucial question left unanswered - what if there is no matched services being offered by a desired business partner? In this situation, such a process has to be negotiated and created. Unfortunately the negotiation and the manual transformation of the negotiation outcome into a workflow model can be very resource consuming in terms of human, time and cost [7]. In fact, it is the computer-aided negotiation approach for processdriven $\mathrm{B} 2 \mathrm{~B}$ e-business that has been largely neglected by the research community. In order to address and respond to the challenge, the next section of the paper introduces the concepts of workflow technology and negotiation. It then identifies three key operational elements found in process negotiation related to workflow technology. Lastly, it explains the integration of IT-supported negotiation services into an overall crossorganisational workflow collaboration (COWCO) supporting framework.

\section{ESSENTIALS OF WORKFLOW TECHNOLOGY AND NEGOTIATION}

For the purpose of clarity, business collaboration discussed in this paper is confined to two business partners but not any particular two.

\section{A. Workflow Essentials}

According to Workflow Management Coalition (WfMC)'s definition, a workflow is "the automation of a business process, in whole or part, during which documents, information or tasks are passed from one participant to another for action, according to a set of procedural rules" [8]. One of the key purposes of workflow is to keep business logic and the underlying application implementation separate, which results in a desired level of flexibility. Although being separated, they are still linked in the form of activities. An activity-based workflow is a workflow that is centred on a set of activities that someone (or something) has to do [9]. Most commercial products and open source projects adopt this model, e.g., IBM's WebSphere MQ Workflow and Enhydra's Shark. The popularity of activitybased workflow is also reflected in the adoption of activity as 
one of the basic building blocks found in mainstream process definition languages, such as IBM's WSFL, WfMC's XPDL and BPMI's BPML [10].

In this paper, interaction points of collaboration processes are modelled as a series of interface activities. Each collaborative message is passed through a pair of interface activities located in both partners' workflows, namely message sending and receiving activities [11]. They are represented by their activity labels followed by either the letter ' $s$ ' or ' $r$ ', meaning sending and receiving, in a pair of square brackets. Collectively, they are known as interface activities. The set of all interface activities of one partner form an interface process. Whenever a message sending activity is reached in a process, it sends a message to the partner and the successor activity will then be triggered. As for a message receiving activity, when it is reached, it is suspended until the right message comes from the other collaboration process. To preserve privacy only interface activities within a process are exposed thus visible to relevant business partners. It is assumed that the direction of a message is in line with each partner's role in a particular business collaboration, e.g. requesting for a Commercial Invoice will not happen from both collaborating partners.

\section{B. Negotiation in A Nutshell}

The word 'negotiation' is derived from the Latin word 'negociare' - to conduct business. Most negotiation was first understood in the context of business transactions although it has been in a wider context for quite some time. As an approach to alternative dispute resolution, negotiation has been studied and defined in many ways $[12,13,14,15,16]$. Distilled from these definitions, a common set of stages comprising the whole process of negotiation is as follows:

- Two partners each with its own interests come together with the intention of reaching some agreement,

- Through communication, if there are any conflicts between their individual interests, they are identified,

- Partners seek possible options to reconcile the differences through a range of strategies and approaches, such as concession making, contending, problem solving, inaction, withdrawal [17], or exploration of mutual gains [18],

- If successful, an agreement is reached be it only in favour of one partner's interests or a win-win result.

Throughout a negotiation process, two core principles [19] should be observed:

- Negotiation is a voluntary activity in the sense that either party can break away from a discussion at any time;

- A successful outcome in negotiation is to get what both sides want rather than to win at any cost.

They act as guidelines for mutual gains discovery, concession giving and decision-making activities during a negotiation process.
Generally speaking, two approaches are often found in negotiation practice, namely concession-convergence and mutual gains. The concession-convergence approach earns its name by following such a paradigm: two parties start with standing on opposite sides and approach each other by giving something up with the aim of making a deal [20]. Also known as 'distributive bargaining' [21], early negotiation research was almost exclusively centred around this approach, which was considered as primitive, competitive or even mindless by some scholars and began to fall into disfavour in the wake of the mutual gains approach [20]. Deemed as the founder of the discipline of organisational behaviour, Follett advocates the mutual gains approach [18]. Different from concessionconvergence, it tries to redefine the negotiation as a shared problem to be resolved. Knowledge and resources are pooled and maximum mutual gains are sought after in order to yield greater payoffs to all parties. Walton and McKersie have used the term "integrative bargaining" [21] and Lax and Sebenius have coined the term "creating value" [22] to capture the same idea. As to the choice between the two approaches, neither is necessarily better than the other $[21,22,23]$. The effectiveness of each approach depends on specific application domains. These researchers also encourage an integrative rather than antagonistic relationship between the two approaches.

As a complicated human centric process, negotiation covers strategic, behavioural and operational aspects. With the concern of this paper in the operational aspect only, three key elements - namely interests, communication and options - are identified. They are based on, and extended from, Fisher's Seven Elements Framework [24].

- Interests - individual needs, concerns, goals, hopes and fears that motivate both partners. Commonality and/or conflict can be found when two sets of individual interests are compared.

- Communication - the transfer of messages by speech, writing or other means for the purpose of comparing individuals' interests, which also includes negotiation protocol, i.e. the manner of message exchange.

- Options - ideas about how the parties might meet their interests together, which includes all the necessary alterations to individuals' initial interests as well as any associated concession in order to reconcile any encountered conflicts.

\section{CAPTURING NEGOTIATION WITH WORKFLOW TECHNOLOGY}

In order to provide IT support for workflow-based negotiation, negotiation should be understood and captured within the context of workflow technology. Within the scope of this research project, IT is aimed to support the operational aspect of process negotiation. The captures of the three relevant constituent operational elements of negotiation through workflow technology are considered in this section. 


\section{A. Interests}

When workflow technology is used to automate business processes, relevant business rules and practice preference have been embedded in workflow process definitions. Each individual's interests are expressed in the form of functions of workflow activities and their control and data dependencies in a process definition. In collaboration context, such interests are expressed as desired collaborative messages to be sent and received by individual workflows arranged in particular orders, which are equally reflected by the corresponding interface activities and their control flow dependencies within a partner's workflow as illustrated in Fig. 1.

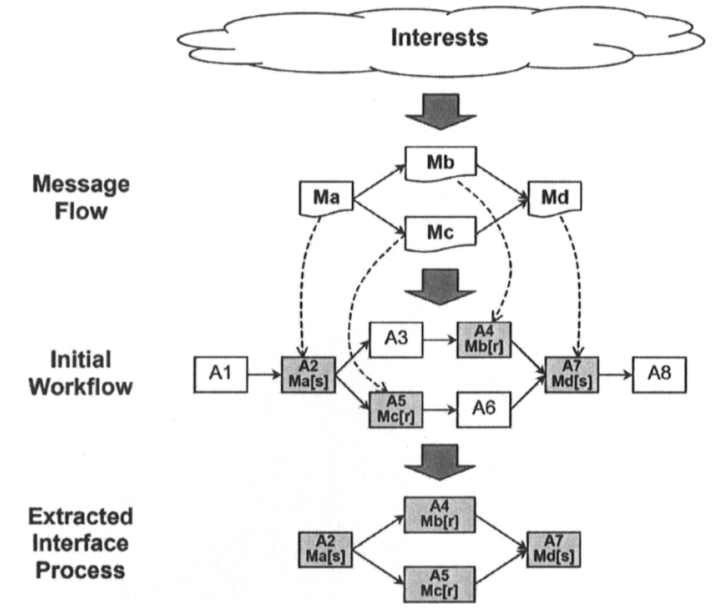

Figure 1. Capturing negotiation interests as an interface process extracted from a workflow.

Between two workflows, common interests lie with the compatibility of the control flows of activities handling collaborative data, which effectively defines a set of process matching criteria. However, whether a set of criteria can truly reflect the commonality is a challenging question. For this reason, the mutual gains approach is adopted in searching for such matching criteria.

Apparently, if the control flows of two processes are exactly the same and the interaction points are properly modelled and matched on both sides, the two processes can certainly collaborate with each other given a proper communication channel. It can be formally defined as:

Definition 1 Absolute Compatibility: Two collaborative workflows are of absolute compatibility if:

- interaction points are modelled as interface activities,

- their interface activities regarding each message are in pairs,

- messages can be delivered through a communication channel,

- the sequence that interface activities appear in both workflows are exactly the same.

However, the cost of achieving such compatibility will be very high under the assumption that the two interface processes must be transformed into exactly the same in order for the collaboration to proceed. A set of less strict and effective matching criteria is desired.

Based on the concept of interface activities, one of the requirements for two workflows to successfully collaborate with each other at runtime is defined as the enact-able compatibility.

Definition 2 Enact-able Compatibility: Two collaborative workflows are of enact-able compatibility if:

- the first 3 conditions of Absolute Compatibility are met and,

- the sequence that interface activities appear in both workflows will not cause message deadlock.

Since the enact-able compatibility requirement exists on the minimum end of the workflow compatibility spectrum, its effectiveness needs to be examined to ensure that it is not too relaxed. Partners $P_{A}$ and $P_{B}$ are the owners of interface processes $A$ and $B$ respectively shown in Fig. 2. Although $A$ and $B$ comply with enact-able compatibility, a satisfactory collaboration cannot be safely guaranteed because activity $A . g[r]$ has to wait for the message $g$ until after activity $B . g[s]$ is completed. The wait for $P_{A}$ could last for days or weeks and unnecessarily delay the completion of $A \cdot g[r]$ thus the whole process $A$, which may not be acceptable for $P_{A}$ from a business perspective despite the fact that no execution deadlock will occur.

A

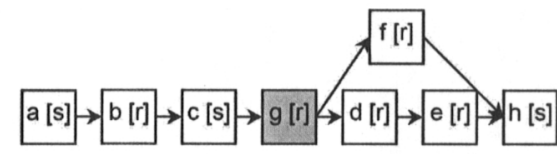

B

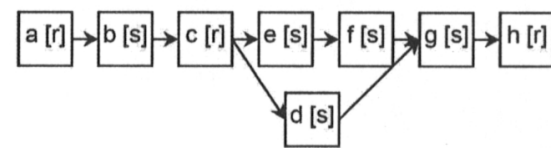

Figure 2. Unnecessary delay might occur despite complying with Enact-able Compatibility.

As a result, before any two business partners can proceed in conducting B2B e-business transactions, their workflows that will be involved in the transactions must be compatible with each other at the business level [25], i.e. they have an agreed sequence(s) of collaborative messages. Ref. [7] approaches bilateral process compatibility through modelling of message sequence and concludes that bilateral collaboration consistency requires trading partners share at least a common message sequence between their message sequence models. Since the model of message sequence can be equally represented by the sequence of each partner's interface activities extracted from an initial workflow, the business collaborative compatibility can therefore be defined as:

Definition 3 Business Collaborative Compatibility: Two collaborative workflows are of business collaborative compatibility if: 
- the first 3 conditions of Absolute Compatibility are met and,

- the two workflows have at least one common sequence of corresponding interface activities.

The justification of the second condition is that corresponding interface activities on a common trace can always be reached by both interface processes in a timely manner, which will leave no collaborative message unattended and thus guarantee a successful collaboration without any deadlock or unnecessary delay.

Acting as process matching criteria, Definition 3 implements the mutual gains approach and highlights the behavioural aspect of processes rather than the structural aspect between the digraph representations of control flows. When two processes do not satisfy Business Collaborative Compatibility, conflicting interest emerges.

Conflicts found in negotiations are the differences between two partners' interests. In workflow collaboration context, they refer to the differences between two interface processes represented by interface activities and their sequences.

As far as two processes are concerned, differences can be found at two levels: structural and behavioural. In this paper they are called structural dissimilarities and behavioural discrepancies respectively. Structural dissimilarities refer to the differences between the two digraph representations of corresponding interface processes, i.e. differences between vertices to edges. Behavioural discrepancies capture process difference by following the control flow and traversing every possible flow trace. According to the common interests matching criteria stated in Definition 3, if there is any difference encountered, it will be a behavioural discrepancy rather than a structural dissimilarity. That is to say Business Collaborative Compatibility tolerates structural dissimilarities to a certain extent as long as they do not cause behavioural discrepancies, which effectively reflects the desired results that the mutual gains approach should bring about.

\section{B. Communication}

The flexibility of the loosely coupled philosophy lies with the autonomous changes of distributed partners. However, it is the inherent uncertainty associated with the autonomy that presents the disadvantage of loose coupling. Therefore, before each deal comes into execution, the deal's two participating workflows need to be exchanged, compared and checked to decide whether they are a matched pair according to the matching criteria described in Definition 3. If not, conflicts need to be revealed. Also, in order to coordinate the communication, a negotiation protocol should be decided for both partners to follow.

Interest comparison for workflows is essentially process comparison. The task is to find an appropriate way of comparing processes in order to effectively reveal behavioural discrepancies. Yeoh, et al [26] propose a method for identifying differences between workflow processes. Despite its achievement of being able to reveal structural dissimilarities that suit its needs, behavioural discrepancies identification is still largely untouched by following such a method. Having been inspired by Krukkert's work reported in [27], a process comparison technique is proposed specifically targeting behavioural discrepancies, in which activity diagram representations are converted into state transition system (STS) digraphs [28].

The negotiation protocol depends on the underlying negotiation approach. In this paper, a combined negotiation approach is adopted, in which the mutual gains approach is used during process comparison to absorb structural dissimilarities and discover hidden common behaviour whilst if any conflict in the form of behavioural discrepancies are encountered, they are coped with by the concessionconvergence approach. In time of conflict, due to the decision towards a concession should be made at real time and based on the result of the assessment between the risk associated with the concession and the risk as a result of the conflict [29], the process of successive decision making should be orchestrated in a manner that at real time only when one partner has no further concession to make with regard to the currently identified conflict, a counteroffer is constructed and passed on to the other partner for consideration. Therefore, the negotiation protocol is named real time sequential protocol.

\section{Options}

Due to the dynamic and changing business environment, there is a good chance that communication reveals conflicting interests between two business partners. According to the negotiation principle mentioned in II, when faced with a conflict, partners should try to figure out how to adjust initial individual interests in order to reconcile the conflict and make a deal. Such adjustments form the reconciliation options. For collaborative workflows, since the interests take the form of interface activities and the associated control flow and data flow, negotiation options are expressed as the adjustments of these flow models.

However, a practical question is raised as "to what extent should the flow models be adjusted?" On the one hand, as a general principle, adjustments must contribute to the reconciliation of the conflict. In workflow collaboration context, behavioural conflicts are caused by differences in control flow. Due to the independent relationship between two parties' interface processes, any adjustment carried out by one party that does not eradicate the conflict will not have any contributing effect on later options available to the other side. It is explained using the example shown in Fig. 3.

Partners $P_{A}$ and $P_{B}$ are the owners of interface processes $A$ and $B$ respectively. If $P_{A}$ takes the option of 'move activity $A . f[r]$ before $A . d[r]$ ' and process $A$ becomes $A_{l}$, there will still be conflict between $A_{1}$ and $B$ and the option available to $P_{B}$ to reconcile the discrepancy will still be the same, i.e. 'move activity B.c[r] before B.f[s]'. That is to say the option of adjustment $\left(A \rightarrow A_{l}\right)$ is not a contribution to resolving the conflict. Therefore, if $P_{A}$ decides to concede, it must fully 


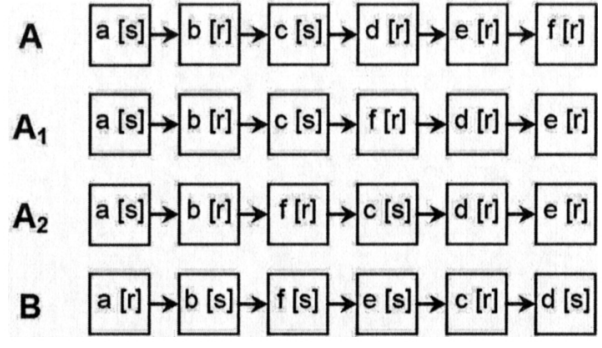

Figure 3. Effectiveness of process reconciliation options.

concede with respect to the current conflict, i.e. 'move $A . f[r]$ before A.c [s]', which would result in process $A_{2}$.

On the other hand, given the causal relationships between activities, the adjustment should not cause any negative side effect on the hosting process, which could lead to some other conflicts. This means that any adjustment shall not only target where the discrepancy occurs, but also where the common trace ends. For processes with only sequential structures, these two types of target position are structurally continuous. However for processes with parallel structures they may turn out to be structurally discontinuous. Since a detailed description of the discontinuous scenario is beyond the scope of this paper, a simple example, as shown in Fig. 3, is used to briefly explain the two-tailed nature of control flow adjustment. Given the two interface processes $A$ and $B$, in order for $P_{A}$ 's reconciliation option to satisfy control flow compatibility requirement, activity $A . f[r]$ should be brought forward but not before the last common activity $A . b[r]$. Otherwise, a new conflict would occur due to A.f[r]'s overshooting.

When the two-tailed control flow adjustment is structurally discontinuous, a safe option is to join all the discontinuous candidate target positions. However it implies that more stringent changes to the initial process are required. In order to work out a more accurate reconciliation option, relevant control flow connectivity needs to be examined. That is to say if no control flow dependency is imposed from a candidate target position, there is no need to include such a position for the purpose of process adjustment, which, as a result, preserves the initial workflow semantics to the maximum level.

Control flow driven adjustments aim to reconcile any behavioural discrepancy without causing any new problem. Relevant connectivity testing tries to contain the changes within a necessary range so that the adjustment has as little impact as possible on the hosting process. Combining both measures, the minimum effective option can be defined, which summaries the requirements of reconciliation options.

Definition 4 Minimum Effective Option: given two unmatched collaborative workflows, minimum effective options refer to adjustments in a hosting workflow with minimum impact on control flow dependencies that can effectively reconcile the currently identified discrepancy.

By following the concession-convergent approach, every reconciliation option may implicate certain concessions. It is important that the concessions are fully captured and presented to human users to enable them to make informed decisions.

As far as workflow adjustments are concerned, concessions exist, again, on two layers - control flow and data flow. Due to the adjustment of interface activities and their sequences, control flow concessions are inevitable. For data flow, whether there will be any implied concession depends on whether the control flow adjustment will cause any data flow conflict. Should any data flow conflict occur, it must be brought to the human users' attention before relevant decisions are made [11].

\section{INTEGRATING IT-SUPPORTED NEGOTIATION INTO CROSS-ORGANISATIONAL WORKFLOW COLLABORATION (COWCO) SUPPORTING FRAMEWORK}

The architecture of the cross-organisational workflow collaboration (COWCO) supporting framework is shown in Fig. 4. For each partner, relevant services include the Collaboration Interface, existing Workflow Management System, the Negotiation and Reconciliation Services, and the Agreement Fulfilment Services. Communication Channels are linking the two service stacks representing each partner.

The services associated with collaboration negotiation and reconciliation can be seen on the build-time half of Fig. 4 whilst the agreement fulfilment services are on the run-time half. In the figure, objects with solid grey borders denote conventional WfMS components. The Collaboration Interface serves as user interfaces between human users and other services. It also coordinates the overall progress from build time through to run time. At build time, the Interface Process Extractor extracts interface processes from initial business processes. The Negotiation and Reconciliation Services

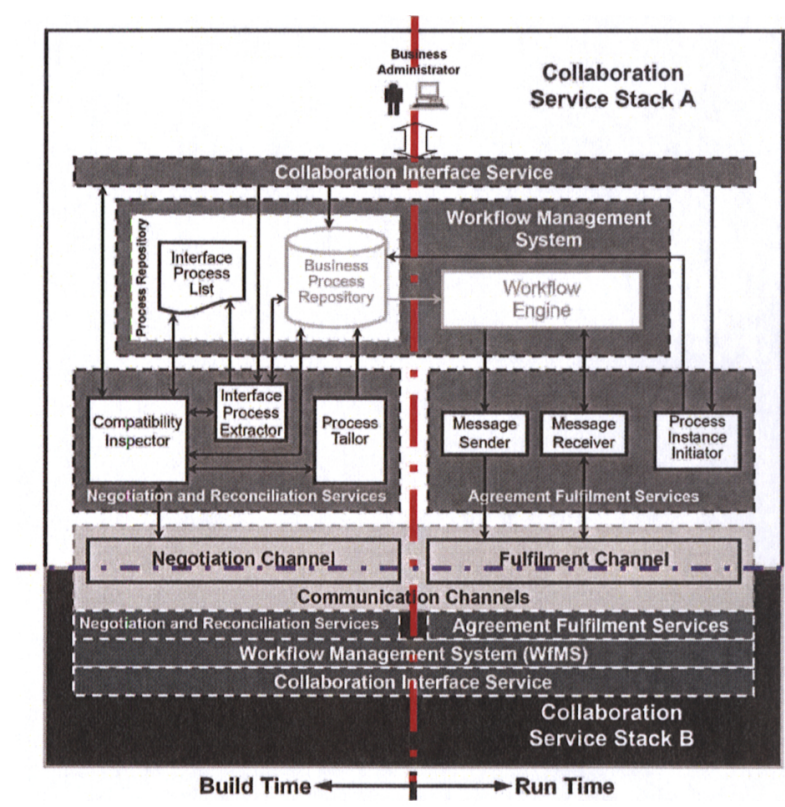

Figure 4. Architecture of cross-organisational workflow collaboration (COWCO) supporting framework. 
provide services for interface process comparison, compatibility checking, discrepancy detection, reconciliation formulation and process tailoring. The Negotiation Channel, acting as a mediator, is used for exchanging intermediate interface processes as negotiation offer/counteroffers. The Message Sender and Receiver of the Agreement Fulfilment Services are attached to a conventional workflow engine. They pass collaboration messages back and forth through the Fulfilment Channel. To test the key concepts, the framework is implemented by following a client/server paradigm with collaboration support services attached to conventional WfMSs on both partners' sites as clients and collaboration mediation services on a server. To ensure the server can pass messages from a Message Sender to the appropriate Message Receiver, an advanced event triggering mechanism is developed based on a blackboard system [30].

\section{CONCLUSIONS}

Recognising the importance of facilitating successful business collaboration between business partners in the form of their existing workflows within a distributed environment, appropriate IT supports are needed at both collaboration build time and runtime. However, only by following a classic service-oriented paradigm, i.e. service discovery followed by service execution, is not able to cope with the situation where there is no existing process-driven service provided by a desired business partner. Therefore, IT support for business process negotiation is required to minimise the potential high cost and demanding resources to reach an agreement and subsequent implementation. Three key operational elements of negotiation are identified and captured within the context of workflow technology. The negotiation services are integrated into an overall cross-organisational workflow collaboration (COWCO) supporting framework and implemented as part of the COWCO-Guru application prototype. It is envisaged that the integrated build-time business process negotiation and the runtime distributed workflow enactment services could further facilitate and automate current B2B e-business practice to meet the increasing complexity and automation needs demanded by the e-business community.

\section{REFERENCES}

[1] RosettaNet, "RosettaNet Business Dictionary, RNBD v2.1" $<$ http://www.rosettanet.org/RosettaNet/Rooms/DisplayPages/LayoutInitia 1?Container=com. webridge. entity.Entity\%5BOID\%5B6EDB5FE87F69D 411BD84009027E33DD8\%5D\%5D>. (accessed 5 May 2005), 2002.

[2] W.M.P. van der Aalst. "Interorganizational Workflows: An Approach based on Message Sequence Charts and Petri Nets," Systems Analysis Modelling - Simulation, 34(3): pp335--367, 1999.

[3] L. Biegus and C. Branki, "InDiA: a Framework for Workflow Interoperability Support by Means of Multi-Agent Systems," Engineering Applications of Artificial Intelligence, 17 (7) pp825-839, 2004.

[4] K.A. Schulz, and M.E. Orlowska, "Facilitating Cross-Organisational Workflows with a Workflow View Approach," Data \& Knowledge Engineering, 51 (1) pp109-147, 2004.

[5] F. Leymann, D. Roller, and M.-T. Schmidt, "Web services and business process management," IBM Systems Journal, vol 41, NO 2, pp198-211, 2002.
[6] A. Wombacher and M. Rozie, "Evolution of Workflow Similarity Measures in Service Discovery," in Proceedings zur Konferenz im Rahmen der Multikonferenz Wirtschaftsinformatik, Lecture Notes in Informatics (LNI), ISBN: 3-88579-174-9, pp 57 - 71, 2006

[7] A. Wombacher, Decentralized establishment of consistent, multi-lateral collaborations, $\mathrm{PhD}$ Thesis at Technical University Darmstadt, Facultiy of Informatics, 2005.

[8] Workflow Management Coalition, "Terminology \& Glossary, Technical Report WFMC-TC-1011,"Workflow Management Coalition, 1999.

[9] F. Guillaume, "Trying to Unify Entity-based and Activity-based Workflows,"

http://www.zope.org/Wikis/DevSite/Projects/ComponentArchitecture/Try ingToUnifiyWorkflowConcepts $>$. (accessed 3 Jul 2005)

[10] R. Shapiro, "A Comparison of XPDL, BPML and BPEL4WS," $\mathrm{http}: / /$ www.ebpml.org/A_Comparison_of_XPDL_and_BPML_BPEL.doc >. (accessed 6 Jul 2005), 2001 .

[11] P.W.H Chung and X. Chen, "Reconciling and Enacting CrossOrganisational Workflow for B2B E-Commerce," in L. Fischer, Ed. 2008 BPM and Workflow Handbook Digital Edition v2, pp347-360, 2008.

[12] Longman Dictionary of Contemporary English, Essex England: Pearson Education Limited, 2002.

[13] I.W. Zartman, "Negotiation as a Joint Decision-Making Process," in I.W Zartman, Ed. The Negotiation Process: Theories and Applications, London: Sage Publications, 1977.

[14] W.C. Hamner and G. A. Yukl, "The Effectiveness of Different Offer Strategies in Bargaining," In D. Druckman, Ed. Negotiations: SocialPsychological Perspectives. Beverly Hills, Calif.: Sage Publications, 1977.

[15] P.H. Gulliver, Disputes and Negotiation: A Cross-Culture Perspective San Diego: Academic Press Inc., 1979.

[16] J.S. Rosenschein and G. Zlotkin, Rules of Encounter, MIT Press, 1994.

[17] D.G. Pruitt and P.J. Carnevale, Negotiation in Social Conflict Buckingham: Open University Press, 1993.

[18] M. P. Follett, in H. C. Metcalf \& L. Urwick, Eds. Dynamic administration: The collected papers of Mary Parker Follett, New York: Harper \& Row, 1942.

[19] J. Hiltrop, and S. Udall, The Essence of Negotiation, Prentice Hall, London 1995

[20] J. Z. Rubin, "Models of Conflict Management," Journal of Social Issues, v50 n1 pp33-45, 1994.

[21] R.E. Walton and R.B. McKersie, A behavioral theory of labor negotiations: An analysis a social interaction system. Ithaca, NY: ILR Press, 1965.

[22] D.A. Lax and J.K. Sebenius, The manager as negotiator: Bargaining for cooperation and competitive gain. New York: Free Press, 1986.

[23] L. Tracy and R.B. Peterson, "A behavioral theory of labor negotiations How well has it aged?," Negotiation Journal, 2, pp93-108, 1986.

[24] R. Fisher, The 7 Elements of Negotiation. < http://www.pon.harvard.edu/hnp/theory/tools/7elements. shtml>. (accessed 1 May 2008)

[25] J. Yang and M. Papazoglou, "Interoperation Support for Electronic Business,". Communications of the ACM June Vol. 43, No. 6, pp39-47, 2000.

[26] M.L. Yeoh, P.W.H. Chung, C.J. Anumba, A. El-Hamalawi, I.A. Motawa "Process change identification using workflow specification matching," in Proceedings of the Tenth Americas Conference on Information Systems, New York, New York, August 2004.

[27] D. Krukkert, "Matchmaking of ebXML business processes, Technical Report IST-28584-OX_D2.3_v.2.0," openXchange Project, Oct 2003.

[28] X.Chen and P.W.H. Chung, "A simulation-based difference detection technique for bottom-up process reconciliation," in Proceedings of 9th International Conference on Enterprise Information Systems (ICEIS 2007), Funchal, Madeira - Portugal, 12-16 June 2007.

[29] J.R. Hicks, The Theory of Wages, London: Macmillan and Co., 1932

[30] D. Corkill, "Blackboard Systems," AI Expert 6(9) pp40-47, 1991. 